Published online 2017 April 13.

Abstract

\title{
Outlines Imaging of Salivary Glands
}

\section{Maryam Mohammadzadeh ${ }^{1, *}$}

${ }^{1}$ Assistant Professor, Department of Radiology, Division of Neuroradiology, Amiralam Hospital, Tehran University of Medical Sciences, Tehran, Iran

"Corresponding author: Maryam Mohammadzadeh, MD, Assistant Professor, Department of Radiology, Division of Neuroradiology, Amiralam Hospital, Tehran University of Medical Sciences, Tehran, Iran. E-mail: m-mohammadzadeh@sina.tums.ac.ir

Received 2016 December 21; Accepted 2017 February 08.

\section{Abstract}

In this regards first of all cross sectional anatomy of salivary glands and different diagnostic modalities such as ultrasound, CAT scan, conventional MRI and nuclear studies will be discussed. Advanced MRI modality such as DCE (dynamic susceptibility enhanced) imaging will be introduced. Then various pathologies of salivary glands including infectious, benign and malignant neoplastic processes and their different imaging characteristics will be mentioned in detail along with high quality images. At the end a diagnostic algorithm for appropriate management of patients with salivary gland lesion will be presented which is very helpful for both clinicians and radiologists especially for residents.

This is an abstract presented in the 33rd Iranian congress of radiology (ICR) and the 15th congress of Iranian radiographic science association (IRSA). 